\title{
Neuropsychiatric manifestations associated with systemic lupus erythematosus: A case report and selective review of literature
}

\author{
Udayan Bhaumik ${ }^{1}$, Hemendra Singh ${ }^{2, *}$ \\ ${ }^{1}$ Junior Resident, ${ }^{2}$ Assistant Professor, Dept. of Psychiatry, Ramaiah Medical College, Bangalore, Karnataka, India
}

*Corresponding Author: Hemendra Singh

Email: hemendradoc2010@gmail.com

\begin{abstract}
Introduction: Systemic lupus erythematosus (SLE) is an autoimmune condition affecting multiple organ systems including central nervous system. Its aetiology lies in abnormal antibody formation and complement activation. Neuropsychiatric manifestations are known to occur in patients with SLE, and vary from psychosis, depression, anxiety to cognitive impairment. Here we report a case of depression with psychotic symptoms and suicidal ideations associated with SLE.

Case Summary: We present the case of a 30-year-old female who presented with irritability, crying spells, feeling of guilt, referential and persecutory ideations along with suicidal ideations. She was diagnosed with severe depression with psychotic symptoms associated with SLE. She improved over a period of 6 weeks with Quetiapine 300mg along with symptomatic management of underlying medical condition. Conclusions: Depression with psychotic symptoms is not an uncommon presentation of SLE. It needs comprehensive management and multidisciplinary approach. Hence physician has to be sensitized regarding neuropsychiatric manifestations of SLE for improving the morbidity and mortality associated with underlying depression.
\end{abstract}

Keywords: SLE-Depression-Psychosis.

\section{Introduction}

Systemic lupus erythematosus is an autoimmune condition affecting the connective tissue of multiple organ systems. It results in complement activation due to production of autoantibodies against a variety of targets including double-stranded deoxyribonucleic acid (dsDNA), ribonucleic acid-binding proteins (RBPs), and phospholipids. ${ }^{1}$ Various environmental, hormonal, and genetic factors have been implicated in causation of this disease. It is nine times more common in women. ${ }^{2}$

The first description of mental changes in lupus was reported by Kaposi in 1872. Despite many advances, CNS lupus often remains misunderstood. Neuropsychiatric manifestations are seen in about $60 \%-70 \%$ cases of systemic lupus erythematosus. ${ }^{3}$ Seizures, peripheral neuropathy or mononeuropathy, headache and transverse myelitis are known among them. Exclusive presentation with psychiatric symptoms is relatively uncommon. ${ }^{4}$ Cognitive impairment (80\%), depression (up to $40-50 \%$ ) and anxiety (10\%) are well-known in Systemic lupus erythematosus. Psychotic symptoms are least frequently described.

Currently, no single clinical, laboratory, neuropsychological, and imaging test can differentiate neuropsychiatric systemic lupus erythematosus from nonneuropsychiatric systemic lupus erythematosus with similar neuropsychiatric manifestations. Here we report a young adult female presenting with psychotic symptoms with depression during a flare of systemic lupus erythematosus.

\section{Case Report}

A 29-yr. old female, homemaker presented with intermittent episodes of irritability, anger episodes and reduced sleep on and off for 3 years. There was no significant family history of mental illness, although there was history of autoimmune disorders in the family, namely in her father who suffered from antiphospholipid antibody syndrome. She had been diagnosed with systemic lupus erythematosus and antiphospholipid antibody syndrome 13 yrs. back. She was on treatment with Prednisolone $4 \mathrm{mg}$ once in the morning, Azathioprine $50 \mathrm{mg}$ morning dose and Hydrochloxychloroquine $200 \mathrm{mg}$ in the morning when first seen. She had suffered from various complications like arthralgia, vasculitis and pleural effusion during illness flares in the past. She had been admitted with acute bronchitis 1 month before index admission. She had also experienced multiple episodes of depression with suicidal ideations in the last 10 years with no response to previous pharmacological treatment. She was not on any psychotropics at the time of seeking consultation. Mental status examination revealed depressive features with mood congruent psychotic symptoms which were related to her physical illness, namely systemic lupus erythematosus. She had also been experiencing delusions of guilt related to her perceived disability from the illness with persecutory auditory hallucinations on same theme.

Patient was investigated for complete blood count where neutrophils were high, and Lymphocytes and Haemoglobin were low (Table). Liver function and renal function tests were normal. C3 and C4 complement levels were also within normal limits. She had elevated prothrombin time, activated partial thromboplastin time and international normalised ratio (Table). Magnetic resonance imaging of brain was normal. Autoimmune profile showed elevation in anti-dsDNA, PCNA, Nucleosome and Beta-2 glycoprotein antibodies. Patient was started on Quetiapine and Clonazepam. At the time of discharged she was on Quetiapine $50 \mathrm{mg}$ morning dose with $200 \mathrm{mg}$ sustained release preparation at night with Clonazepam 0.5 mg 1-0-2. At follow up she was put on Quetiapine $300 \mathrm{mg}$ at night and maintaining well. 
Table 1: Investigation reports

\begin{tabular}{|l|c|c|}
\hline \multicolumn{1}{|c|}{ Parameters } & Values & Reference Value \\
\hline Neutrophils & $85.1 \%$ & $40-80$ \\
\hline Lymphocytes & 10.5 & $20-40$ \\
\hline Haemoglobin & 11.0 & $12-15$ \\
\hline Prothrombin time & 26.9 & $11-16$ \\
\hline $\begin{array}{l}\text { Activated partial } \\
\text { thromboplastin time }\end{array}$ & 42.2 & $30-40$ \\
\hline $\begin{array}{l}\text { International } \\
\text { Normalised Ratio }\end{array}$ & 2.34 & $1-1.3$ \\
\hline Anti ds-DNA & 141.29 & $<100$ \\
\hline B2 Glycoprotein IgM & 66.7 & $<20$ \\
\hline Anti-PCNA & positive & \\
\hline Anti-nucleosome & positive & \\
\hline
\end{tabular}

\section{Discussion}

Various neuropsychiatric manifestations among patients with SLE might also be associated with concurrent steroid therapy. In our case, the patient was on oral steroids for a long duration, with no increase in dosing of steroids in past 1 year. This implies psychotic symptoms to be primarily due to the disease.

There is a strong genetic predisposition to SLE, with 35 genes associated with the occurrence of the disease being identified (including interleukin receptor associated kinase 1, HLA DR2 and HLA DR3), with a heritability of $0.66 .{ }^{5}$ The pathogenesis of neuropsychiatric systemic lupus erythematosus is multifactorial. Activation of various inflammatory cytokines, autoantibodies, and immune complexes result in vasculopathy, cytotoxic and autoantibody-mediated neuronal injury. ${ }^{6}$ The most common microscopic brain finding appears to be non-specific micro vasculopathy from complement activation and antiphospholipid antibodies. ${ }^{6}$ Disruption of blood brain barrier appears to be central to its pathogenesis. Endothelial cells responsible for maintenance of the blood-brain barrier may have altered function in the presence of elevated levels of Intracellular adhesion molecule-1 during flare-ups. ${ }^{5}$ It has also been implicated in corticosteroid-induced neuropsychiatric symptoms in these patients. Anti-double stranded Deoxy ribonucleic acid antibodies have been found to cross react with subunits NR2 and NR3 of the N methyl D aspartate (NMDA) glutamate receptors triggering excitotoxicity and mediating apoptosis through caspase-3 mediated pathways. Anti-ribosomal $\mathrm{P}$ glycoprotein is also known to contribute to psychosis. ${ }^{7}$ Our patient had positive anti-dsDNA titres when she presented with psychotic symptoms. In patients with SLE the neurobiological mechanism underlying the association appears unclear. Magnetic resonance imaging changes in neuropsychiatric presentations mainly include hyperintense lesions in periventricular and subcortical white matter. ${ }^{8}$ Hippocampal atrophy has been seen in chronic cases. ${ }^{9}$

Absence of primary diagnostic criteria for primary neuropsychiatric SLE makes diagnosis difficult unless high index of suspicion is maintained. Factors predisposing to depression in this illness are appearance concerns, loss of self-esteem, inadequate pain control, poor coping, perceived severity of disease, social isolation, concealment of symptoms, and major life events. Depression is more likely in the setting of poor quality of medical care. Diabetes mellitus, vascular disease, unemployment, and low levels of education are also found to be associated with depression in isolated studies. ${ }^{10}$ Suicidal risk is high in these patients. ${ }^{11}$ Psychotic spectrum disorders in SLE is however not wellstudied. Acute psychosis appears to be commonest, with a good prognosis in majority of cases. Magnetic Resonance Imaging findings in psychosis are usually normal and nonspecific, as was seen in our case. ${ }^{12}$

\section{Conclusions}

This case teaches us that antipsychotics can be used to target psychotic symptoms even if it clearly due to a systemic illness. High index of suspicion for neuropsychiatric symptoms for at-risk individuals or patients with history of systemic lupus erythematosus is also required. In any case of neuropsychiatric systemic lupus, it is important to rule out secondary causes (e.g.: corticosteroid-induced) for the psychosis. Exclusion of neuro-infections, the commonest cause of morbidity and mortality.in such cases is mandated. Treatment is based on accurate diagnosis of specific neuropsychiatric manifestations. Assessment of the degree of SLE mediated systemic disease activity is also required. ${ }^{(3)}$ Complement levels, dsDNA, complete blood count and urine analysis help in finding out the disease activity. Presence of neuropsychiatric manifestations suggests cautious use of steroids and other anti-inflammatory drugs. Thus, it suggests a poor outcome ${ }^{13}$ unless aggressively and judiciously managed.

\section{Acknowledgment: Nil.}

\section{Conflicts of Interest: Nil.}

\section{References}

1. Brendan M. Giles B M and Susan A. Boackle S A. Linking complement and anti-dsDNA antibodies in the pathogenesis of systemic lupus erythematosus. Immunol Res. 2013;55(0):10 21.

2. Ward MM. Prevalence of physician-diagnosed systemic lupus erythematosus in the United States: Results from the third national health and nutrition examination survey. $J$ Women's Health (Larchmt).2004;13:713-718.

3. Brey RL, Holliday SL, Saklad AR, Navarrete MG, HermosilloRomo D, Stallworth CL, et al. Neuropsychiatric syndromes in lupus: Prevalence using standardized definitions. Neurol. 2002;58:1214-1220.

4. Bhoi S, Kumar M, Lamba Singh BM, Gupta BB. Primary Psychiatric Presentation in Systemic Lupus Erythematosus. $J$ Indian Acad Clin Med. 2001;2(4).

5. Ramos P S, Brown E E, Kimberly R P, Langfeld C D. Genetic Factors Predisposing to Systemic Lupus Erythematosus and Lupus Nephritis. Semin Nephrol. 2010;30(2):164-176.

6. Kivity S, Agmon-Levin N, Zandman-Goddard G, Chapman J, Shoenfeld, Y Neuropsychiatric lupus: a mosaic of clinical presentations Medicine. 2015;13:43. DOI 10.1186/s12916$015-0269-8$ 
7. Nayak R B, Bhogale G S,Patil N M,Chate S S. Psychosis in Patients with Systemic Lupus Erythematosus. Indian J Psychol Med. 2012;34(1):90-93.

8. Sibbitt, W.L Jr Sibbitt, R.R. Brooks W.M. Neuroimaging in neuropsychiatric systemic lupus erythematosus. Arthritis Rheum. 1999;2(10):2026-2038.

9. Appenzeller S, Carnevalle A.D, Li L.M, Costallat L.T, Cendes F. Hippocampal atrophy in systemic lupus erythematosus. Ann Rheum Dis. 2006;65(12):1585-1589.

10. Gupta M. Depression in systemic lupus erythematosus: a systematic review. Int J Students Res. 2015;5(2).

11. Mok C C, Chan K L, Cheung E F C, Yip P S F. Suicidal ideation in patients with systemic lupus erythematosus: incidence and risk factors. Rheumatol. 2014;53:714-721.
12. Huizinga TW, Steens SC, van Buchem MA. Imaging modalities in central nervous system systemic lupus erythematosus. Curr Opin Rheumatol. 2001;13:383-388.

13. Sibley JT, Olszynski WP, Decoteau WE. The incidence and prognosis of central nervous system disease in systemic lupus erythematosus. J Rheumatol. 1992;19:47-52. 